\title{
ISTTOK plasma control with the tomography diagnostic
}

\author{
P. J. Carvalho ${ }^{\mathrm{a}}$, H. Thomsen ${ }^{\mathrm{b}}$, R. Coelho ${ }^{\mathrm{a}}$, P. Duarte ${ }^{\mathrm{a}}$, C. Silva ${ }^{\mathrm{a}}, \mathrm{H}$. \\ Fernandes $^{\mathrm{a}}$ \\ ${ }^{a}$ Associação Euratom/IST Instituto de Plasmas e Fusão Nuclear, Instituto Superior \\ Técnico 1049-001 Lisboa, Portugal \\ ${ }^{b}$ Max-Planck-Institut für Plasma Physik, EURATOM Assoziation, 17491 Greifswald, \\ Germany
}

\begin{abstract}
The presently available processing power in Generic Processing Units (GPU) combined with state-of-the-art programmable logic devices enables the implementation of complex algorithms for plasma diagnostics in a real-time scenario.

A tomography diagnostic based on three linear pin-hole cameras each with eight lines of sight has been developed for the ISTTOK tokamak. The plasma emissivity in a poloidal cross-section is computed locally on a sub-millisecond time scale, using a variant of the Fourier-Bessel algorithm. The output signals are then used for active plasma position control.

The data acquisition and reconstruction system is based on ATCA technology and consists of one acquisition board with integrated FPGA capabilities and a dual-core Intel module running RTAI Linux.

In this paper, the tomographic algorithm and some preliminary results of the real-time plasma position control are presented with a performance benchmarking against other available positioning diagnostics. The algorithm has shown to be accurate and the system has successfully controlled the plasma position during a plasma current reversal.
\end{abstract}

Keywords: ISTTOK, Tokamak, Tomography, Fourier-Bessel, Data Acquisition

PACS: 52.55.Fa, 42.30.Wb, 07.05.Hd

\section{Introduction}

A real-time plasma position control system is required to achieve long duration (up to 250ms), Alternating Current (AC) discharges on the ISTTOK tokamak $\left(R=46 \mathrm{~cm}, a=8.5 \mathrm{~cm}, I_{p}=4 \mathrm{kA}, B_{T}=0.5 \mathrm{~T}\right.$ and $\left.t_{\text {discharge }}=30 \mathrm{~ms}\right)$. Such a system has been in place for some time based only on magnetic field diagnostic data [1]. However, this system has been found to be limited during the current inversion of $\mathrm{AC}$ discharges. The installed tomography diagnostic has been configured to supply additional feedback to the control system. In 


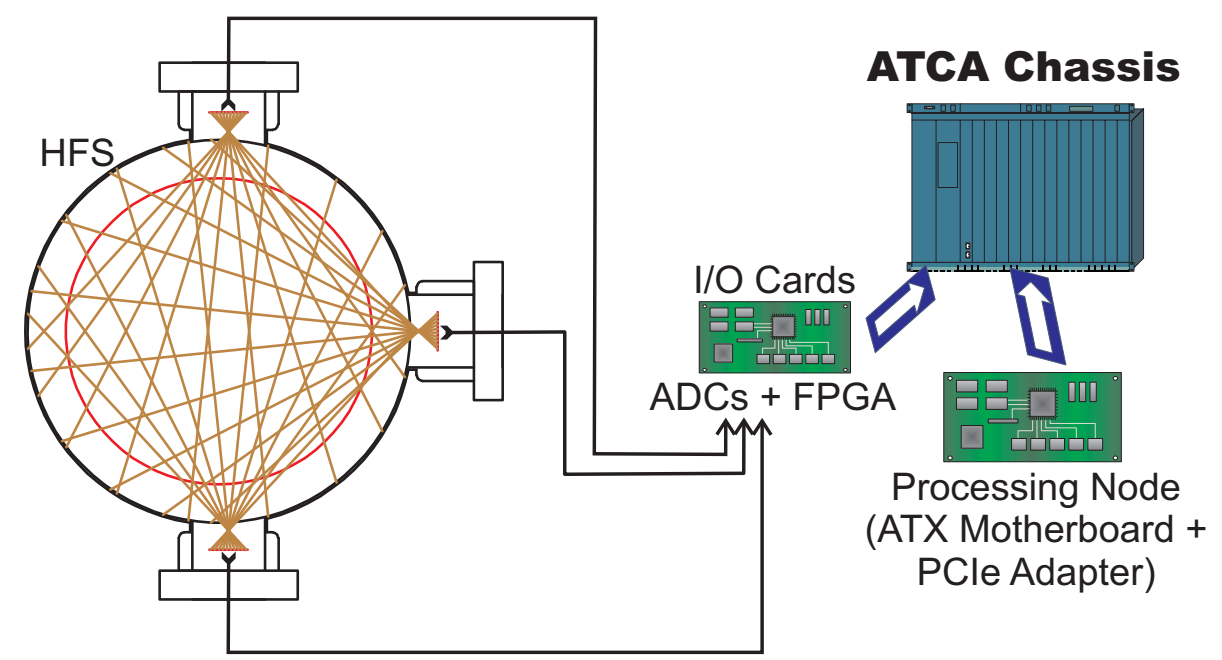

Figure 1: The tomography data acquisition ATCA system installed in ISTTOK. The 24 channels are acquired by the I/O card and relayed to the PC module via the ATCA bus.

this work, the magnetics based control was disconnected and only the tomography based control was employed. This paper presents (i) the Data Acquisition and Reconstruction (DAR) real-time system, based on Advanced Telecommunications Computing Architecture (ATCA) technology [2], (ii) the tomographic algorithm, and (iii) the plasma positioning method. These two can be processed in real-time (one feedback loop in less than $100 \mu \mathrm{s}$ ). Some preliminary results are also presented which indicate that the tomography system is an adequate replacement for the solely magnetic control system.

This paper is organized as follows. In section 2 the hardware setup is described. A discussion on the tomography inversion algorithm is detailed in section 3.2, together with an appropriate algorithm benchmarking to provide a confidence level for the reconstructions. Finally, an example of one AC discharge where feedback position control was assisted by the tomography position estimator is presented in section 4 .

\section{Data Acquisition}

On ISTTOK, a very generic and powerful acquisition hardware is available. In this work, the tomographic signals are acquired on 24 channels of one I/O board. The reconstruction, plasma position calculation and required vertical field coil current determination are performed on the GPU. The interface between the I/O board and the GPU is acomplished by the MARTe platform [3]. One full acquisition, calculation and feedback relay cycle takes less than $100 \mu \mathrm{s}$, allowing the system to act in real-time.

The Data acquisition and reconstruction (DAR) real-time system is based on ATCA technology. A detailed account of the ATCA acquisition system can 
be found in [2].

The DAR at ISTTOK uses two ATCA modules, one is the acquisition board and the other is an interface from a standard PC motherboard to the ATCA bus via the PCI-Express port (see Fig. 1). The acquisition board provides 32 analog input channels. It is an ATCA single width card comprising a main board, a carrier board, isolated ADC modules and a rear transition module (RTM) mechanically connected together.

The main I/O board has a Xilinx XC4VFX60 FPGA [4], providing digital signal processing and a standard independent communications unit. A total of 15 RocketIO multi-gigabit transceivers $(2.6 \mathrm{Gbits} / \mathrm{s})$ connect the FPGA to the ATCA fabric channels, allowing full mesh topologies, plus one connected to the RTM.

Each ADC module consists of one differential analog input with a dynamic range of $\pm 5 \mathrm{~V}, 16$ ENOB (Effective Number Of Bits) resolution at 2 MSamples per Second (MSPS), double pole passive anti-aliasing filter, where the first stage is a $500 \mathrm{kHz}$ third order filter and the second stage is a $180 \mathrm{kHz}$ first order filter, and galvanic isolation.

The RTM consists of 8 galvanically isolated DAC channels, each with one differential analog output, a dynamic range of $\pm 10 \mathrm{~V}, 16$ bit resolution at up to 50 MSPS.

Feedback commands are relayed by optical link from the PC motherboard to the vertical field coil power supply controller. At $921.60 \mathrm{kbaud}$, the serial communication is fast enough to keep up with the acquisition downsampled rate of $10 \mathrm{kSPS}$.

\section{Tomography}

\subsection{Sensors}

The ISTTOK tomography diagnostic consists of 24 photodiode channels, 8 in each of the 3 Hamamatsu silicon photodiode arrays ${ }^{1}$. Each camera has a circular pinhole of $1 \mathrm{~mm}$ at a distance of $10 \mathrm{~mm}$ from the photodiode array, allowing a full coverage of the plasma. No filter is used so each photodiode is mainly measuring visible and ultra-violet light. Each channel is amplified by a transductance amplifier with a gain of $1.8 \times 10^{6}$ with a cutoff frequency of $58 \mathrm{KHz}$.

\subsection{Algorithm}

In plasma tomography, the goal is to find the emissivity function, $g(r, \theta)$, from several line integrated measurements, $f_{L}(p, \phi)$ :

$$
f_{L}(p, \phi)=\int_{L} g(r, \theta) d L
$$

\footnotetext{
${ }^{1}$ These arrays are similar to the current model Hamamatsu S5668 series, which have a spectral response in $300-1100 \mathrm{~nm}$.
} 


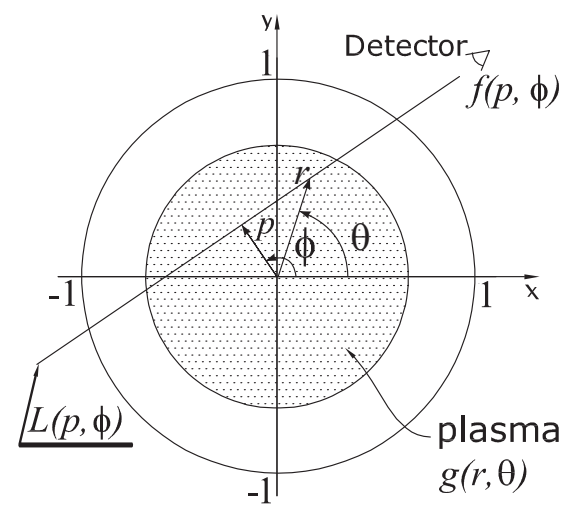

Figure 2: Illustration of one optical sensor.

Assuming that $g$ is a linear combination of some basis functions, it is possible to analytically invert equation (1) [5-8].

In reality, the light is integrated along a cone of sight, rather than a line. It is thus desirable to have an algorithm which accounts for this cone of sight while keeping the calculations simple. The tomographic algorithms used in this work is a generalization of the method presented in [9]. This algorithm has the additional advantage of being applicable to non-circular geometries. However, it can't handle open flux-surfaces, as can be found in the divertor region of a tokamak like JET.

The standard basis function decomposition of $g(r, \theta)$ is

$$
g(r, \theta)=\sum_{n=0}^{N} \sum_{l=0}^{L} a_{n l}^{c} g_{n l}(r) \cos (n \theta)+a_{n l}^{s} g_{n l}(r) \sin (n \theta)
$$

The $N$ and $L$ values for the decomposition are determined from the number of cameras and the number of sensors per camera, respectively, by the Nyquist theorem. They have been set to $N=2$ and $L=2$, which violates the Nyquist theorem for the $N$ as it should be half the number of cameras, but that is compensated by the lower value of $L$. Since there are no $a_{0 l}^{s}$ coefficients, in total 15 basis functions have been used. This means that the emissivity reconstruction will be a linear combination of these 15 basis functions and this limits the possible resolution of the system.

This method can be called Fourier-Generic because $g_{n l}(r)$ can be replaced by any function which presents some level of radial localization with $l$ (in Eq. (2) this index only enters in the radial part).

The numerical integration is accomplished by using a projection matrix, $\mathbf{P}$, which attributes the contribution from each pixel in the reconstruction grid to each sensor value. It is, in fact, a synthetic diagnostic and can be represented as

$$
\vec{f}=\mathbf{P} \vec{g}
$$


where $\vec{g}$ is the 2-D emissivity distribution as a 1-D vector. The projection matrix is thus only valid for a given reconstruction grid and camera setup. Multiplying $\mathbf{P}$ by the mapping of each of the basis functions on the reconstruction grid, one obtains the columns of the contribution matrix, $\mathbf{C}$. This matrix then has the contributions of each basis function to each of the sensors.

Since the integration is done numerically, it is not necessary to restrict the calculation to circular geometry and a generalized $\left(r^{\star}, \theta^{\star}\right)$ grid can be constructed in such a way that, for example, a given $r^{\star}$ corresponds to a radial position of a cross-section of an equilibrium flux surface which could be calculated with a standard code like VMEC [10]. In these generalized coordinates, equation (2) remains exactly the same, but as a function of $\left(r^{\star}, \theta^{\star}\right)$.

With the contribution matrix, it is possible to perform a least-squares fit of the sensor data, $\vec{f}$, to the basis functions using the Singular Value Decomposition (SVD) method [11], where

$$
\vec{f}=\mathbf{C} \vec{a}
$$

and find $\vec{a}$ which are the coefficients that are input to equation (2) to build the final reconstruction.

Note that the contribution matrix pertains to the generalized coordinates as well as the result from the least-squares fit. In order to have a reconstruction in standard $(r, \theta)$ coordinates, it is only necessary to re-map the reconstruction.

The basis functions used in the angular direction come from the Fourier decomposition and are therefore orthogonal. The basis functions used in the radial direction, $g_{n l}(r)$, can be non-orthogonal, so virtually any 1-D function can be used. In this work, the following radial basis functions were tested (see figure 3):

- Radially localized B-splines, just like in [9];

- Radially localized cubic-splines;

- Radially localized Gaussian functions;

- Radially localized triangular function interpolated;

- Radially localized Heaviside functions;

- Bessel functions $J_{n}\left(x_{n l} r\right)$.

Of all these radial basis functions, only the Bessel functions have a dependency on the parameter $n$.

The advantage of using radially localized basis functions lies in the simplicity of the interpretation of the result. It is possible to look at the reconstruction and have a good idea of which basis functions contribute to each radial position. However, the use of non-orthogonal basis functions will, in general, lead to redundant information, which, in turn, can lead to less accurate reconstructions than those obtained with orthogonal basis functions. This effect is visible in the 

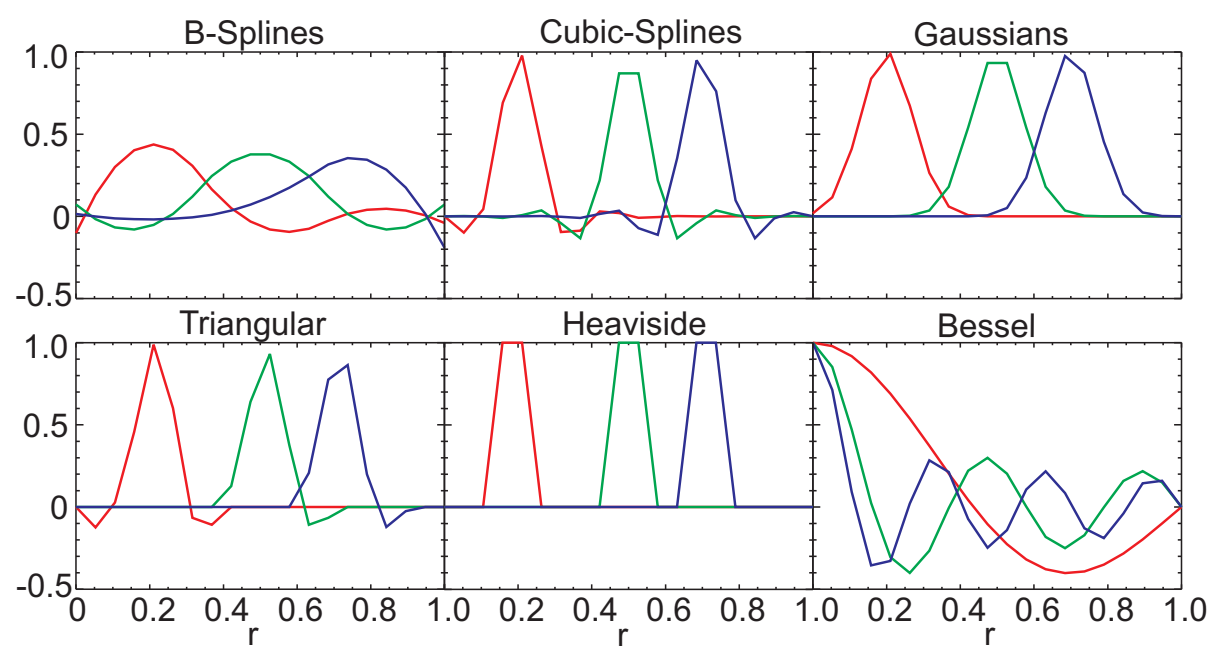

Figure 3: Plot of the basis functions considered in this work. For each type of basis functions, only 3 are shown. In the case of radially localized functions, they are at $r=0.2, r=0.5$ and $r=0.7$.

poor reconstructions obtained with the proposed radial basis functions different from the Bessel functions (Figures 4 and 5).

Typically, the Fourier-Generic method should use flux coordinates according to the imposed magnetic configuration. For the ISTTOK case, given that it operates at low pressure and has a circular limiter, it is expected that the flux coordinates are centered in the geometric center of the vacuum vessel and are circular.

The plasma position is calculated by taking the average center of the emissivity reconstruction.

A standard PID algorithm is employed to calculate the required current on the vertical field coil which acts upon the plasma shifting it to the required position.

This algorithm was implemented as a MARTe module and is running on the GPU routinely calculating the plasma position. The contribution matrix and its SVD, as well as the basis functions, are pre-calculated to minimize online processing time.

\subsection{Algorithm testing and benchmarking}

Several virtual emissivity profiles (phantoms) were tested, of which only two are presented here; a peaked (figure 4) and a hollow profile (figure 5). The error presented in the figures $(\epsilon)$ is the absolute value of the difference between the reconstruction and the phantom, summed over all pixels and normalized to the number of pixels.

Three extra tomographic reconstruction algorithms were introduced for comparison and validation of the Fourier-Generic method described above. These 
extra algorithms are the standard Fourier-Bessel [8], the Maximum Entropy [12] and the Minimum-Fisher [13]. These last two are constrained regularization algorithms, where the regularization (Entropy or the Fisher integral) are maximized or minimized with the constraint that the data must be well represented. The Maximum-Entropy method yields the solution with the maximum likelihood, assuming that the reconstruction tends to a state of maximum entropy. The Minimum-Fisher method presents the smoothest possible solution, which is desired since the plasma is not expected to evolve steep gradients in the radiation between adjacent pixels.

Of all the radial basis functions tested in the Fourier-Generic algorithm, only the Bessel functions yielded consistently reasonable reconstructions. This algorithm has shown to be clearly superior to the standard Fourier-Bessel, as can be seen in figure 4. The Minimum-Fisher algorithm has shown to provide a very good agreement with the original phantoms, so it is the algorithm of choice for offline tomographic analysis. It can't be run as a real-time application due to its iterative nature, which renders it too slow (one reconstruction takes about 1 minute when calculated offline, versus about $40 \mathrm{~ms}$ for the Fourier-Generic).

\subsection{Fourier-Generic with real data}

In this subsection, the plasma position calculated by both the proposed tomographic algorithm and the magnetic diagnostic are shown for a fully preprogrammed discharge.

Shot \#17552 from ISTTOK is a two full-cycle shot, with $1 \mathrm{kA}$ flat-top plasma current with an average density of $1.7 \times 10^{18} \mathrm{~m}^{-3}$ (see figure 6).

Figure 6 shows the temporal evolution of the plasma position for this shot, determined by the magnetics [1] and the average emissivity of the tomographic reconstructions calculated by the Fourier-Generic algorithm using the Bessel functions as radial basis. There is a significant difference between both positions. This difference is due to the disparate nature of the measurements and the neglecting of the external magnetic fields in the calculations. The tomography system measures light emissivity, while the magnetics are sensitive to the plasma current density distribution. The average center of the current may not be the same as the average center of the emissivity, although they should, generally, be close. Also, the algorithm used to calculate the position from the magnetic measurements doesn't take into account the currents flowing through the external field coils. These fields alternate with each current reversal and this effect is visible in the magnetic position of figure 6 . When the plasma current is in it's normal direction (first half-cycle), the magnetics place the plasma inand downwards. On the other hand, when the plasma current is reversed, the magnetics place the plasma in the opposite top- and outwards. The tomography shows the reversed behaviour and with much less amplitude $(<1 \mathrm{~cm})$. Due to assembly constrains, the poloidal coils are not exactly symmetric, which leads to this plasma behaviour and is the main obstacle behind an adequate algorithm adjustment for the necessary correction fields.

Calculating the position from Minimum-Fisher reconstructions, it follows the Fourier-generic algorithm's, but with an amplitude of about $1 \mathrm{~cm}$, while 
the Fourier-Generic positions the plasma much closer to the center. This result validates the Fgen with the Minimum-Fisher algorithm, pointing out that the real-time algorithm (Fgen) doesn't represent the full amplitude of the plasma column's shift in the vessel.

\section{Results}

In this section, a shot with the horizontal plasma position controlled by the tomography diagnostic is presented. The full feedback loop takes $100 \mu$ s so only one of every two samples is used in this loop.

ISTTOK's shot \#23330 was an AC shot with one full cycle. It had an average plasma current of $3.2 \mathrm{kA}$ and a density of approximately $2.6 \times 10^{18} \mathrm{~m}^{-3}$. The shot was configured so as to invert the plasma current at $t=27 \mathrm{~ms}$ and end at $t=51 \mathrm{~ms}$. From the start until $t=4 \mathrm{~ms}$ the vertical field was in preprogrammed mode because of the PRECO [14] (pre-discharge capacitor bank discharge) current which can have undesired effects on the control algorithm. From $t=4 \mathrm{~ms}$ onward, the vertical field was always in feedback mode, where the plasma position was determined by the tomography diagnostic and the plasma position setpoint was at the center of the vessel, $R=460 \mathrm{~mm}$. Given that the tomography data is insensitive to the plasma current direction, the algorithm has no means of determining when it should invert the current in the vertical field coil. This information must be pre-programmed into the algorithm and, to avoid the time the field takes to reverse $(\sim 1 \mathrm{~ms})$ this inversion is pre-programmed to occur at $t=26 \mathrm{~ms}, 1 \mathrm{~ms}$ before the actual plasma current reversal.

Figure 7 shows two reconstructions from this shot. One before the plasma current reversal and the other after it. The plasma exhibits a hollow profile in both instances. The asterisk in the figure specifies the plasma position calculated from the reconstruction.

In figure 8 , the horizontal position of the plasma is shown as determined by four different diagnostics. The tomography diagnostic which is under trial, a set of eight Mirnov coils, a set of two electrostatic probes and a cosine coil. The plasma current and vertical field coil's current are also shown.

The magnetic field diagnostic determines the plasma position by approximating it to a current filament [1]. The electrostatic probes, measuring floating potential, are positioned on the low field side (LFS) and the high field side (HFS) of the device, at the vessel equatorial plane. The floating potential radial profile at the probes radial location $(r=75 \mathrm{~mm})$ is very steep $(E r \sim 50 \mathrm{~V} / \mathrm{cm})$, being its variation roughly linear with radius in a small region around the probe position $(\sim 1 \mathrm{~cm})$. The difference between the signals measured at the LFS and HFS is therefore directly proportional to the horizontal plasma position for small displacements $(<1 \mathrm{~cm})$.

The cosine coil is similar to a rogowsky coil but with variable winding density, proportional to the cosine of the poloidal angle and the winding direction reverses after $180^{\circ}$ to give the negative contribution. Its signal is proportional to the horizontal position of the average plasma current. After the plasma in- 
version, this signal is reversed, but it has been normalized by the plasma current sign in Figure 8.

It is apparent that all the positions agree among each other, at least qualitatively. The tomographic position is always close to the setpoint, with a few oscillations in the first half-cycle, but relatively steady in the second. The Mirnov coil diagnostic yields a plasma position consistently offset to the LFS and null when the plasma current is below $500 \mathrm{~A}$, which renders it inoperative during the plasma reversal. The maximum cross-correlation of the tomographic position with the position from the Mirnov coil, during the first half-cycle is of 0.8 , which shows that both measurements agree. However, during the second half-cycle, the maximum cross-correlation is of only 0.45 which shows that the two signals are not agreeing although both show a tendency towards the HFS.

The cosine coil yields a plasma position very consistent with the tomographic one except just after the current reversal where it shows the plasma moving far to the LFS, but then returning to near the axis and again showing the tendency to the HFS that both previous diagnostics have shown. The maximum crosscorrelation of this signal with the tomographic position is, for the first half-cycle, of 0.75 and, for the second, 0.83 , meaning that both signals agree very well with each other.

The ES probes yield a plasma position close to the vessel axis, but with a slightly offset to the LFS during the whole shot. The maximum cross-correlation of the signal with the tomographic one is of 0.75 for the first half-cycle and 0.56 for the second. Again, a good agreement is found during the first half-cycle, but not during the second, in spite the fact that the ES probes also show the tendency toward the HFS.

During the current reversal, the Mirnov coil diagnostic is inoperative, the cosine coil and the ES probes show the plasma moving from the HFS to the center of the vessel, which is the same as the tomographic position, although this one overshoots the center but quickly returns there.

The current on the vertical field coil $\left(I_{V}\right)$ is already negative when the plasma current is reversed which helps to keep the plasma close to the center just after the plasma current reversal. However, the plasma also moves upward (not shown in figure) and this upward movement is causing the plasma to remain displaced. At $t=34 \mathrm{~ms}$, the vertical position of the plasma is centered and the horizontal position also becomes centered. This is proof that a horizontal field control is desireable to maintain the plasma in place during the whole discharge.

In general, the tomography diagnostic is correctly assessing in which direction the plasma column is displaced as is shown by high cross-correlation factors. However the other three diagnostics considered don't agree in the absolute value of the position of the plasma and so it is not possible to be certain of the absolute position calculated by the tomographic system.

\section{Potential Upgrades}

From the previous section, it is obvious that control over the vertical plasma position is required. It is part of a planned upgrade and the currently imple- 
mented algorithm already takes it into consideration. This means that, once the power supply for the horizontal field is online, there will be no impact on the calculation time.

A reconstruction optimization test has been undertaken to infer what would be the optimum setup for cameras and cameras resolution considering the port restrictions of ISTTOK. This test used several phantoms and a sweep on the number of sensors per camera, assuming that the camera dimension was fixed. Figure 9 presents such a sweep for a phantom similar to the reconstructions presented in figure 7. In general, a configuration with 20 to 30 sensors per camera shows a good performance, not always the best, but very close. The most straightforward improvement, with little impact on the overall diagnostic setup, would be to replace the existing photodiode arrays.

An ideal upgrade would be the inclusion of more cameras, covering the vessel from all sides. If processing time, number of acquisition channels and machine access were not a problem, it would be possible to insert up to 18 Hamamatsu s5668 arrays with 16 photodiodes each. These would be positioned roughly at $r=85 \mathrm{~cm}$ and their pinholes would be at $r=80 \mathrm{~cm}$. Amplification and digitization of the data would be performed in-vessel, just behind the arrays, to avoid contamination of the signal in the cables. Due to time constraints, a maximum of 60 channels could be used for plasma position control. This would ensure a more reliable reconstruction and more accurate estimation of the position.

\section{Summary}

In this work, the tomography diagnostic at ISTTOK was presented as a real-time diagnostic which controls the horizontal position of the plasma column. Each feedback loop has a $100 \mu$ s period during which the acquired and downsampled signals are passed to the PC, the tomographic reconstruction is calculated, its average center is determined, the PID algorithm sets the current to shift the plasma column to the required location. This current value is passed to the vertical field coil power supply which imposes the magnetic field that shifts the plasma horizontally.

While the standard Fourier-Bessel algorithm [8] relies on the assumption that radiation is integrated along lines of sight, the tomographic reconstruction algorithm used in this work considers the integration along cones of sight, which is a more realistic view. It can also be used in non-circular configurations and can take any one-dimensional function as radial basis functions, hence the proposed name Fourier-Generic.

It has been shown that this diagnostic yields a good agreement with other diagnostics in determining the plasma displacement. However, the absolute value of the displacement away from the axis of the vessel may not be correct. This could be improved by having more sensors, but it would have an impact on the calculation time.

The system has managed to successfully control a plasma current inversion, even without vertical position control. The inclusion of a vertical position con- 
trol system would obviously allow a much better control of the overall plasma position and obtain a much more stable plasma. Such inclusion is planned and is already implemented in the algorithm running on the PC, which means that the time it will take to perform this extra control function will be the same as the present system.

Acknowledgments This work has been carried out within the framework of the Contract of Association between the European Atomic Energy Community and "Instituto Superior Técnico". Financial support was also received from "Fundação para a Ciência e Tecnologia" in the frame of the Contract of Associated Laboratory.

[1] D. F. Valcárcel et al., Fast Feedback Control for Plasma Positioning With a PCI Hybrid DSP/FPGA Board, IEEE Transactions on Nuclear Science, 53, 2006, pp838-844

[2] A. J. N. Batista et al., ATCA digital controller hardware for vertical stabilization of plasmas in tokamaks, Rev. Sci. Instrum. 77, 10F527 (2006), DOI:10.1063/1.2221906

[3] André Neto et al., Linux real-time framework for fusion devices, Fusion Engineering and Design, 84, 2009, pp 1408-1411

[4] http://www.xilinx.com/support/documentation/virtex-4.htm

[5] A. M. Cormack, Representation of a function by its line integrals, with some radiological applications, Journal of Applied Physics, 34, 1963, pp2722

[6] A. M. Cormack, Representation of a function by its line integrals, with some radiological applications. II, Journal of Applied Physics, 35, 1964, pp2908

[7] Ling Wang and Robert S. Granetz, A simplified expression for the Radon transform of Bessel basis function in tomography, Review of Scientific Instruments, 62, 1991, pp842-843

[8] Ling Wang and Robert S. Granetz, An analytical expression for the Radon transform of Bessel basis function in tomography, Review of Scientific Instruments, 62, 1991, pp1115-1116

[9] G.T.A. Huysmans et al., MHD stability of optimized shear discharges in JET, Nuclear Fusion, 39, 1999, 11, pp1489-1507

[10] S.P. Hirshman and W.I. van RIJ, Three-dimensional free boundary calculations using a spectral Green's function method, Computer Physics Communications, 43, 1986, pp143-155

[11] William H. Press, Saul A. Teukolsky, William T. Vetterling and Brian P. Flannery, Numerical Recipes in C, The Art of Scientific Computing, Second Edition, Cambridge University Press, 1992 
[12] K. Ertl et al., Maximum Entropy based reconstruction of soft X-Ray emissivity profiles in $W^{7}$ - $A S$, Nuclear Fusion, 36, 1996, pp1477-1488

[13] M. Anton et al., X-ray tomography on the TCV tokamak, Plasma Physics and Controlled Fusion, 38, 1996, pp1849-1878

[14] C.A.F. Varandas et al., Engineering aspects of the tokamak ISTTOK, Fusion Technology, 29, 1996, pp105 


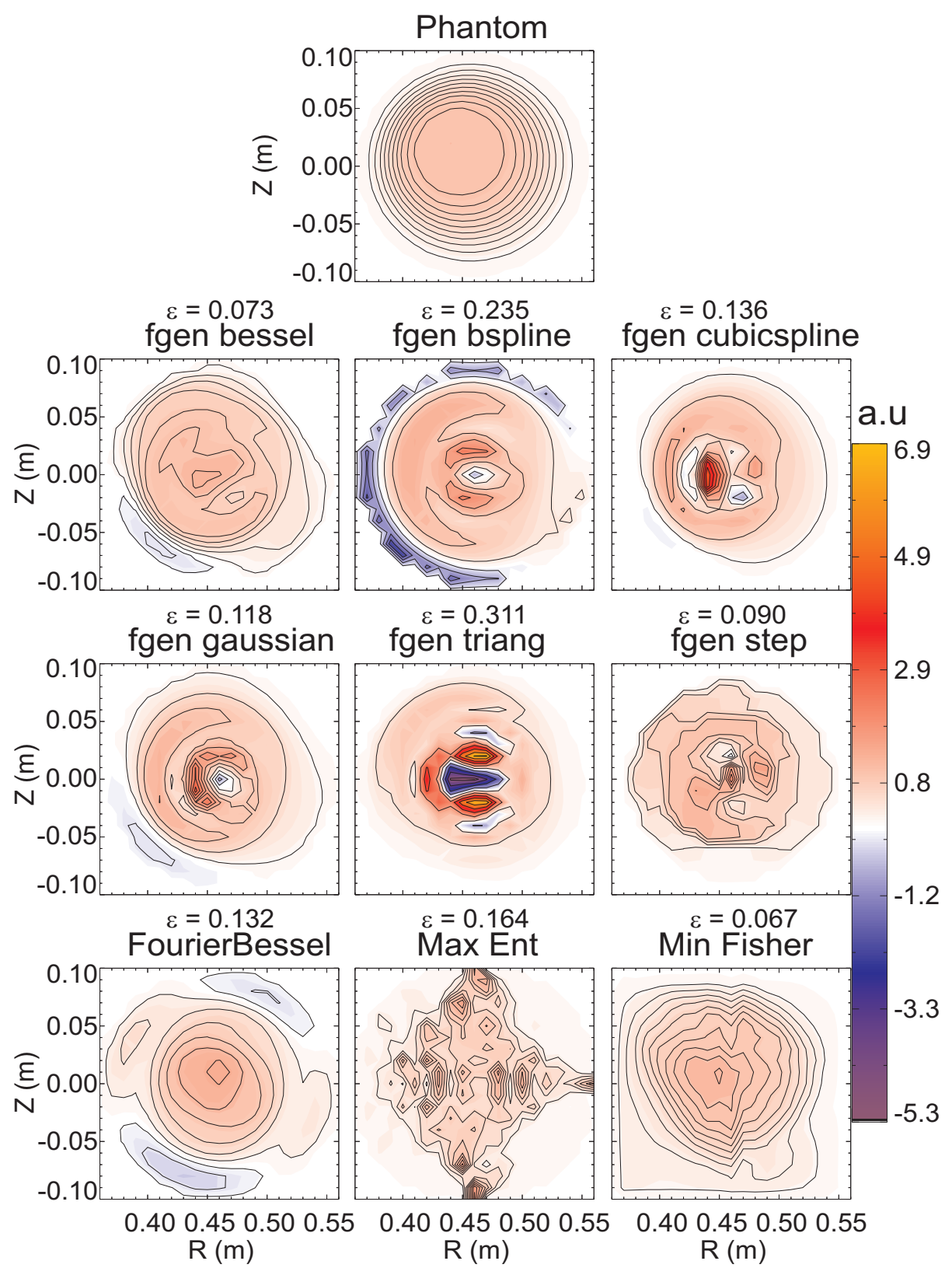

Figure 4: Reconstructions of the off-centered peaked phantom with $3 \%$ Gaussian noise added on the signals. Only the fgen Bessel, fgen Step and Minimum Fisher methods yield an acceptable reconstruction, but all of them display some undesirable artifacts. The remaining fgen methods hint at the general direction of the shift. The Fourier-Bessel is only slightly shifted and the Maximum Entropy, although also hinting at the shift, it also displays too many artifacts. 


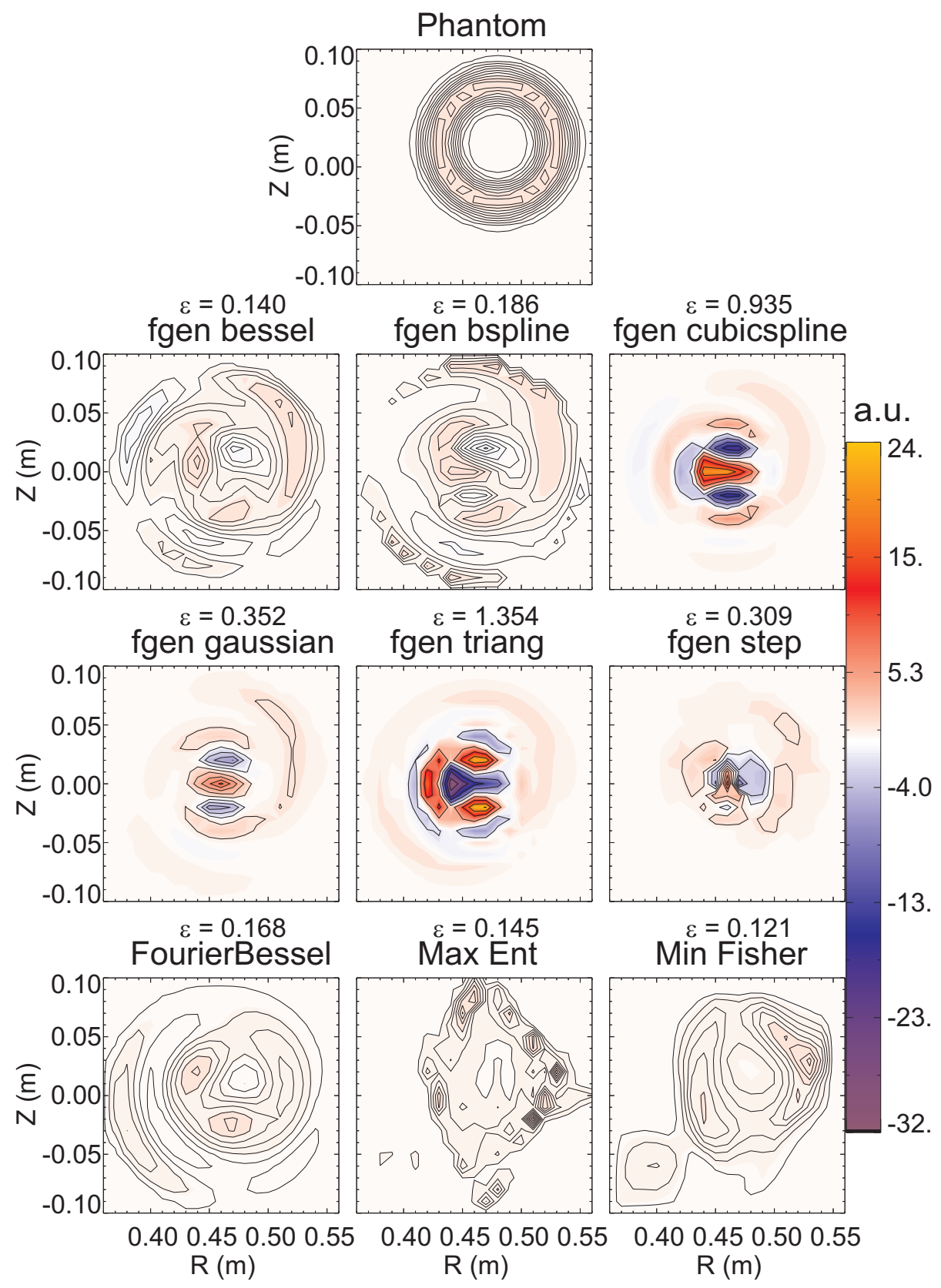

Figure 5: Reconstructions of the ring phantom. Only the Minimum Fisher method reconstructed the ring, but still yielded an unacceptable error. The fgen-Bessel and BSpline, the Fourier-Bessel and the MaxEnt reconstructions hint at the ring shape. All other reconstructions yielded too high an error to be considered acceptable. 


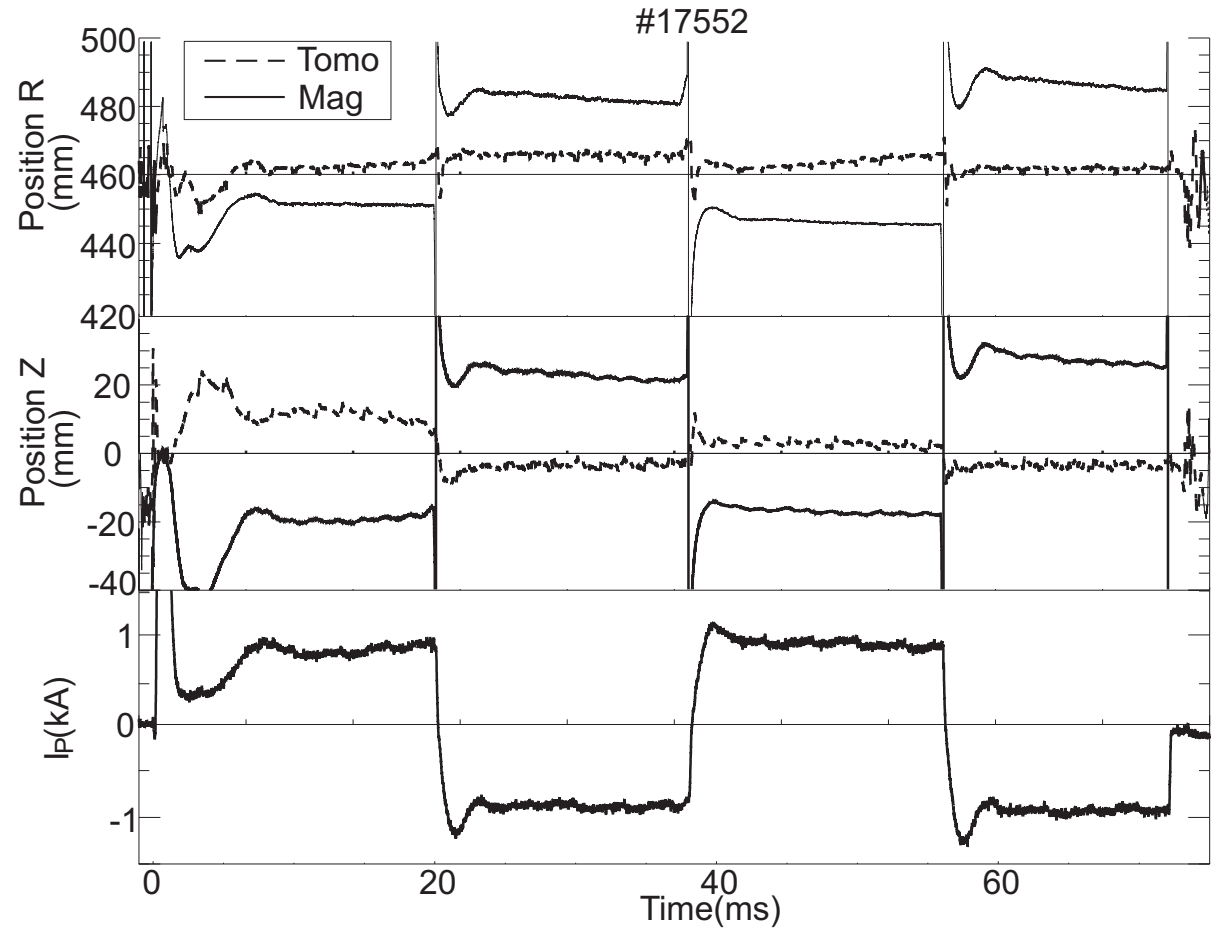

Figure 6: Plasma position for shot \#17552 determined by the average center of the tomography reconstruction and the magnetic diagnostic's current filament.

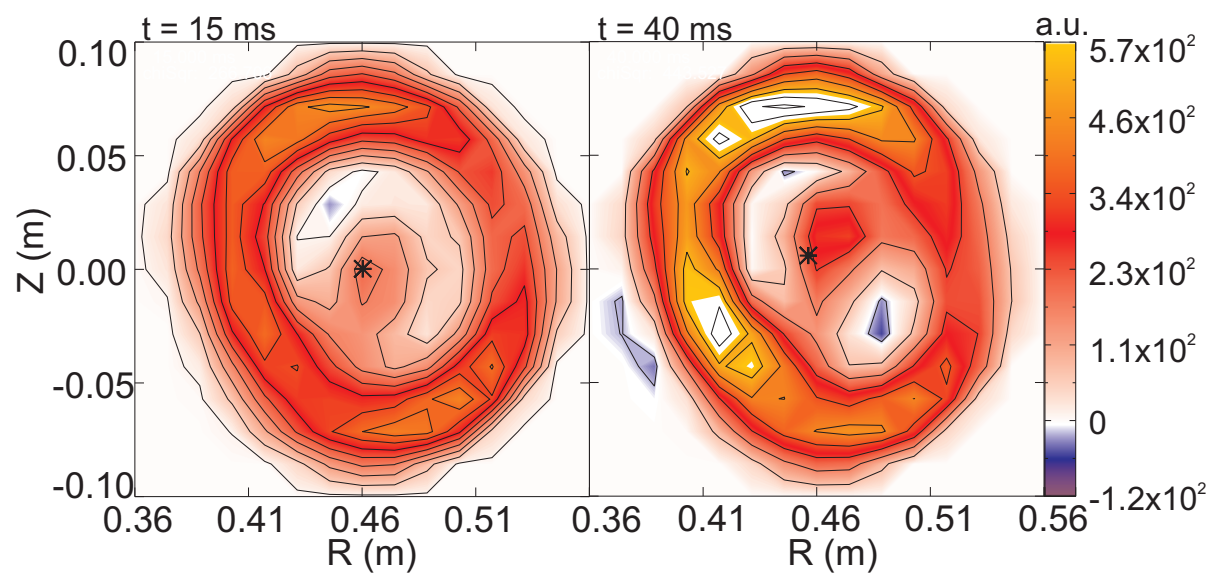

Figure 7: Two tomographic reconstructions from shot \#23330, for $t=15 \mathrm{~ms}$ and $t=40 \mathrm{~ms}$. 


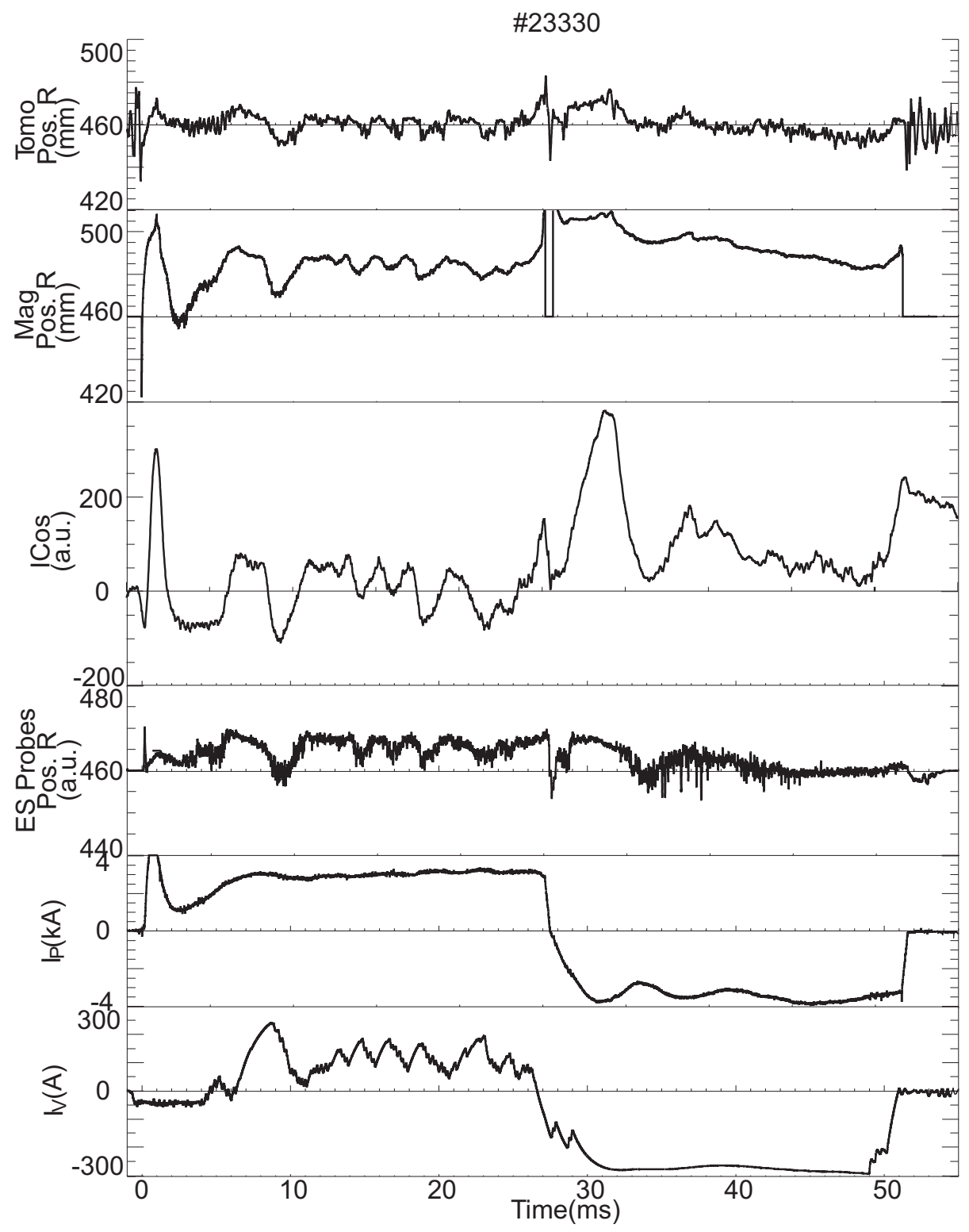

Figure 8: Shot \#23330's horizontal plasma position, determined by tomography, Mirnov coils (Mag), cosine coil (ICos) and electrostatic (ES) probes. The plasma current $\left(I_{P}\right)$ and vertical field coil current $\left(I_{V}\right)$ are also shown. 


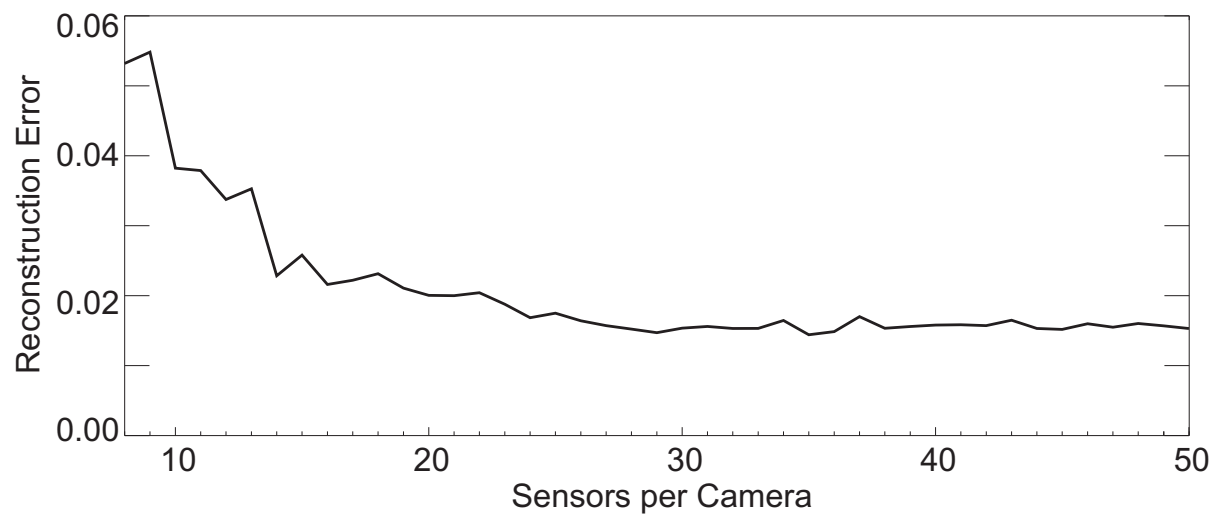

Figure 9: Error in the reconstruction of virtual data versus the number of sensors for a phantom with a hollow profile and a peak near the center. 